\title{
La Dirección de Operaciones de Servicios (DOS)
}

(1) Franklin Ríos Ramos

\section{RESUMEN}

Este artículo consiste en una revisión bibliográfica de los aportes teóricos que han ido surgiendo en el ámbito de los servicios. El primer objetivo de este artículo es determinar e estado actual de las investigaciones en la Dirección de Operaciones de Senvicios, y el segundo es proponer una guía en este tema para futuras investigaciones.

Palabras Clave: Dirección de Operaciones de Servicios. empresas de servicios.

S ervice Operations Management (SOM) ABSTRACT

This paper is a literature review about theoretical approaches that have emerged in the service field. The first purpose is to determine the state of the question in the research of Service Operations Management. The second is to offer a guide for future researches.

Key words: Service Operations Management. Service companies.
I N T R O D U C C I Ó N

A pesar de que el estudio e investigación de la Dirección de Operaciones de Servicios (DOS) llevan ya casi más de un cuarto de siglo, todavía no se tiene cubierta las bases de esta disciplina. Sin embargo las economías de muchos países están basadas en la operación de empresas de servicios, por ello es relevante estudiar este tema, especialmente en países que estamos en vías de desarrollo. Con esta investigación no se ha podido encontrar estudios teóricos o empíricos sobre este tema de origen latinoamericano, las investigaciones más cercanas a nuestra realidad son de origen español, pero también se ha podido detectar que existen en general grandes vacíos y temas pocos estudiados por los diversos estudios hechos y publicados en las revistas más relevantes.

REVISIÓN TEÓRICA

Comenzaremos la revisión teórica citando las principales revistas científicas donde se tocan los temas relacionados con la Dirección de Operaciones: (1) Decisión Sciences, (2) Harvard Business Review, (3) Internacional Journal of Operations and Production Management (4) International Journal of production Research (5)Journal of Operations Management (6) Management Science (7) Production and Operations Management y otros.

De estas revistas según Gonzáles Zamora et al. (2003), el $6.8 \%$ de los artículos son dedicados específicamente a la Dirección de Operaciones de Servicios (dato al 2002). También en este estudio los autores tratan los temas más relevantes tocados en estas revistas:

1. Introducción y problemática de los servicios

2. Estrategias y Objetivos de Operaciones en Servicios

3. Diseño del Subsistema de Operaciones en Servicios

4. Planificación, Programación y Control de Servicios

5. Cadena de Suministro y Logística

Para esta revisión teórica utilizaremos esta clasificación, para los grupos temáticos de la Dirección de operaciones en servicios (DOS), que desarrollaremos en el presente artículo.

Introducción y problemática de los servicios

No es necesario comprobar que en el Mundo la tendencia hacia la industria sin humo (servicios) esta en notable crecimiento, y ya tiene una importancia vital en la economía de algunos países, pues su actividad nacional se esta centrando en está. Son muchos los sectores de servicios que

(1) MBA de la Universidad Carlos III, España. Ingeniero Industrial, Pontificia Universidad Católica del Perú Profesor de la Facultad de Estudios de la Empresa de la Universidad Peruana de Ciencias Aplicadas, UPC E-mail: pcadfrio@upc.edu.pe 
actualmente hacen uso las personas, los siguientes son los 14 sectores relacionado a servicios según Gonzales Zamora et al (2003):

1. Transporte

2. Distribución comercial

3. Sanidad (servicios de salud),

4. Turismo, ocio, cultura y deporte

5. Telecomunicaciones

6. Entidades financieras y de seguros

7. Servicios a empresas

8. Servicios públicos y sin animo de lucro

9. Mantenimiento y reparación

10. Producción y suministro de energía en general

11. Servicios profesionales

12. Correos y mensajería

13. Educación (enseñanza)

14. Servicios personales

La Dirección de Operaciones no tiene que ver solamente con manufactura, las empresas de servicios están descubriendo cada vez más que las ideas y prácticas de la gerencia de operaciones ya son un insumo esencial para su control de costos, mejora del sistema y niveles de servicio al cliente. Operaciones se define como "un medio por el cual los insumos de recursos se combinan, reforman, transforman y se separan para crear resultados útiles (bienes y servicios)".

\section{Concepto de Servicio}

Desde un punto de vista comercial, tanto bienes como servicios ofrecen beneficios o satisfacciones; y son productos. La visión estrecha de un producto dice que es un conjunto de atributos, tangibles e intangibles, físicos y químicos, reunidos en una forma especial. La visión amplia, dice que es un conjunto de atributos, tangibles e intangibles, que el comprador puede aceptar para satisfacer sus necesidades y deseos. Así pues, en el sentido más amplio, todo producto tiene elementos intangibles, independientemente de lo que se produzca en la fábrica.

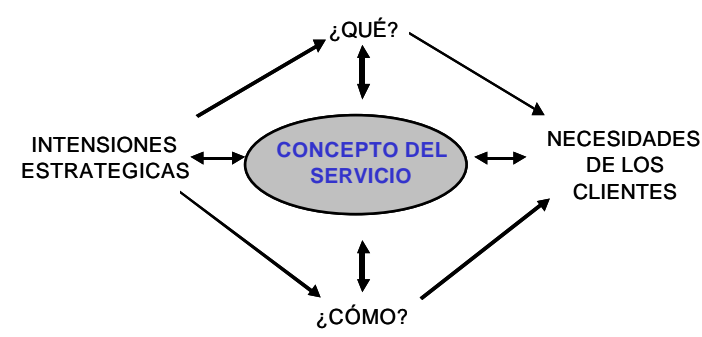

Figura 1. Concepto de servicio Fuente: Goldstein et al.(2002)
El concepto de servicio, tiene hasta hoy muchas propuestas y pocas investigaciones científicas sobre su concepto, por ejemplo, según Stanton (1992) los servicios son "todas aquellas actividades identificables, intangibles, que son el objeto principal de una operación que se concibe para proporcionar la satisfacción de necesidades de los consumidores". Otra definición similar es la que plantea Kotler (1993) que: "un servicio es todo acto o función que una parte puede ofrecer a otra, que es esencialmente intangible y no da como resultado ninguna propiedad. Su producción puede o no puede vincularse a un producto físico." . Pero nosotros nos quedamos con la propuesta de Goldstein et al.(2002), donde su definición la esquematiza y generaliza de la manera que muestra la figura 1.

El concepto de servicio no sólo se define como el ¿Qué se da? y ¿Cómo de da?, sino como la integración de estos elementos con la estrategia de la empresa y las necesidades de los clientes. Esta definición será de mucha utilidad para el diseño del servicio.

De lo anterior se deduce que las organizaciones de servicios son aquellas que no tienen como meta principal la fabricación de productos tangibles, que los compradores vayan a poseer permanentemente; es decir, la compañía está vendiendo el servicio como núcleo central de su oferta al mercado, que esta alineado con la estrategia de la compañía y las necesidades de los clientes.

Características diferenciadoras de los Servicios Se han sugerido varias características para ayudar a distinguir bienes y servicios. Es la combinación de estas características la que crea el contexto específico en que debe desarrollar Dirección de Operaciones en una organización de servicios. Según Arias Aranda (2001), De Burgos etal. (2003), Rivera y Huete (1997), Pancorvo (2001)y Fitzsimmons y Fitzsimmons (1998), las características más frecuentemente establecidas de los servicios son:

\section{a. Intangibilidad}

Los servicios son esencialmente intangibles. Con frecuencia no es posible gustar, sentir, ver, oír u oler los servicios antes de comprarlos. Se pueden buscar de antemano opiniones y actitudes; una compra repetida puede descansar en experiencias previas, al cliente se le puede dar algo tangible para representar el servicio, pero a la larga la compra de un servicio es la adquisición de algo intangible. 
De lo anterior se deduce que la intangibilidad es la características definitiva que distingue productos de servicios y que intangibilidad significa tanto algo palpable como algo mental. También la falta de propiedad es una diferencia básica entre una industria de servicios y una industria de productos, porque un cliente solamente puede tener acceso a utilizar un servicio determinado. El pago se hace por el uso, acceso o arriendo de determinados elementos.

\section{b. Perecibilidad}

Los servicios son susceptibles de perecer y no se pueden almacenar. Por otra parte, para algunos servicios una demanda fluctuante puede agravar las características de perecibilidad del servicio. Las decisiones claves se deben tomar sobre qué máximo nivel de capacidad debe estar disponible para hacer frente a la demanda antes de que sufran las ventas de servicios. Igualmente hay que prestar atención a las épocas de bajos niveles de uso, a la capacidad de reserva o a la opción de políticas de corto plazo que equilibren las fluctuaciones de demanda.

Esto dificulta las prácticas tradicionales de Dirección de Operaciones relacionadas con la Planificación y Producción de productos, especialmente en los relacionados a capacidad e inventarios. Tomando el ejemplo del artículo de De Burgos et al. (2003), los hoteles suelen operar con unos niveles de ocupación de su capacidad productiva muy inferiores a los que serían deseables en términos de eficiencia y eficacia. Comparémoslos con una empresa manufacturera, esta última tiene mayor flexibilidad para hacer frente a aumentos en la cantidad demandada de bienes y puede, en general, aumentar los turnos de producción para hacer frente a ella, pero no lo puede hacer de manera inmediata los hoteles.

\section{c. Heterogeneidad}

Con frecuencia es difícil lograr estandarización de producción en los servicios, debido a que cada "unidad", por lo que se genera el fenómeno de la personalización. Prestación de un servicio puede ser diferente de otras "unidades". Además, no es fácil asegurar un mismo nivel de producción desde el punto de vista de la calidad. Asimismo, desde el punto de vista de los clientes también es difícil juzgar la calidad con anterioridad a la compra. Este equilibrio debe traducirse en una serie de procedimientos encaminados a estandarizar las operaciones "Back Office".
Implicación directa o indirecta de los clientes Con frecuencia los servicios no se pueden separar del cliente. Una consecuencia de esto es que la creación o realización del servicio puede ocurrir al mismo tiempo que su consumo, ya sea este parcial o total. Los bienes son producidos, luego vendidos y consumidos mientras que los servicios se venden y luego se producen y consumen por lo general de manera simultánea. Esto tiene gran relevancia desde el punto de vista práctico y conceptual, en efecto, tradicionalmente se ha distinguido nítidamente funciones dentro de la empresa en forma bien separada, con ciertas interrelaciones entre ellas por lo general a nivel de coordinación o traspaso de información que sirve de input para unas u otras; sin embargo, aquí se puede apreciar más una fusión que una coordinación, el personal de producción del servicio, en muchos casos, es el que vende y/o interactúa más directamente con el cliente o usuario mientras éste hace uso del servicio ("consume"). Recordemos que en la manufactura por lo general el personal de producción y el proceso productivo en sí, no suponen interacción directa con el consumidor.

\section{d. Intensidad de capital}

La norma habitual en el sector de servicios es la baja intensidad de capital del proceso, que se encuentra, en la mayoría de los casos poco mecanizado y, en un número aun menos de ocasiones, automatizado. En los últimos tiempos esta circunstancia ha mejorados debido a los sistemas de información y al desarrollo de las comunicaciones. Sin embargo aun existen numerosas operaciones que debe realizarse manualmente y no requieren de tal calificación, por ejemplo en los hoteles serán las operaciones de limpieza y cafetería o restaurante.

Estrategias $y$ operaciones en servicios Se considera las cuestiones sobre objetivos estratégicos de DOS: clasificación de servicios, aspectos estratégicos de la calidad, productividad de servicios, expansión de servicios o nuevas tecnologías en servicios.

\section{Clasificación de servicios}

Antes de clasificar a los servicios es necesario de terminar los componentes de este, lo que en la literatura se llama: "Paquete de servicios", utilizando las propuesta de Arias Aranda (2001) y Fitzsimmons y Fitzsimmons (1998):

a. Instalaciones de Apoyo (supporting facilities): son los recursos físicos imprescindibles 


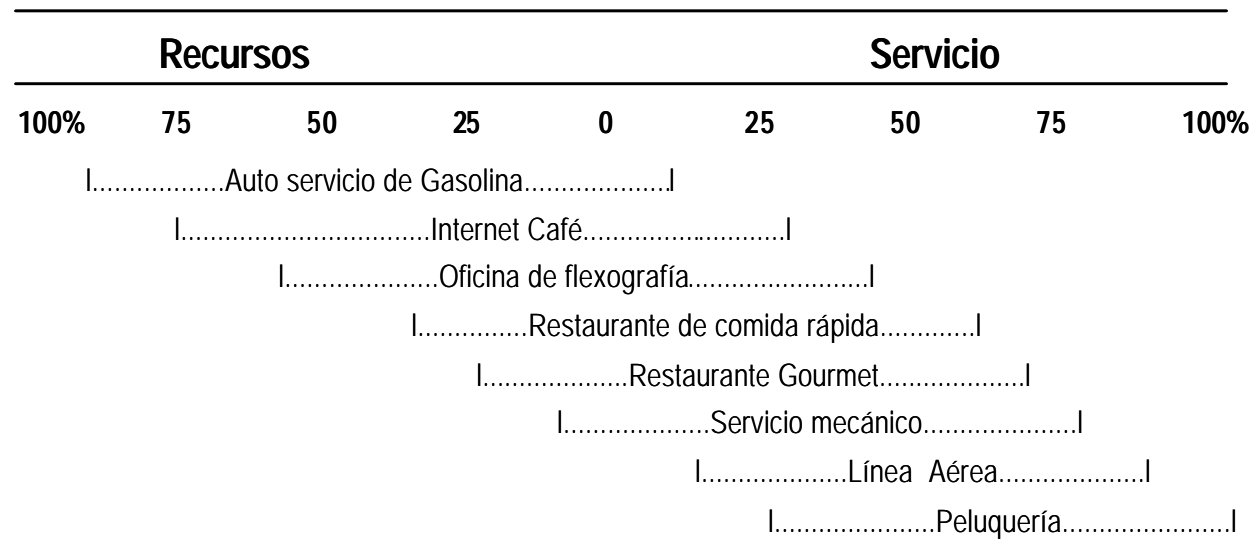

Figura 2. Clasificación de los servicios por el contenido de sus recursos Fuente: Adaptado de Fitzsimmons y Fitzsimmons (1998)

para la prestación del servicio, como el edificio del hospital, el avión, el tren, las instalaciones del hotel, etc.

b. Bienes facilitadores del servicio (Facilitating goods): es el conjunto de bienes que son parte del servicio, que son comprados, arrendados o prestados para la realización plena del servicio. Por ejemplo los palos de golf, los repuestos del automóvil, etc.

c. Servicios Explícitos (Explicit services): beneficios directamente observables por los sentidos y consistentes en las características esenciales o intrínsecas del servicio. Por ejemplo disfrutar del jugar en campo de golf, curarse después de la operación, el excelente funcionamiento del auto después de su reparación, etc. d. Servicios Implícitos (Implicit services): beneficios psicológicos características extrínsecas del servicio percibidas por el cliente. Por ejemplo el estatus que conlleva jugar golf en un campo determinado, la privacidad del trato en un banco, etc.

Es posible clasificar a los servicios por su contenido de recursos ( bienes e instalaciones) de apoyo a éste, como puros y mixtos (ver figura 2).

Otra clasificación de los servicios se da por el grado de interacción y personalización; y el grado de intensidad de mano de obra (ver figura 3).

La primera mide hasta que punto el cliente puede influir de manera personal a la naturaleza del

\section{Grado de Personalización}

\begin{tabular}{|c|c|c|}
\hline & Bajo & Alto \\
\hline Grado de & $\begin{array}{l}\text { Factoría de Servicios: } \\
\text { - } \quad \text { Líneas Aéreas } \\
\text { - } \quad \text { Transporte por carretera } \\
\text { - } \quad \text { Poteles } \\
\quad \text { arques de ocio }\end{array}$ & $\begin{array}{l}\text { Taller de Servicios: } \\
\text { - Hospitales } \\
\text { - } \quad \text { Reparación de automóviles } \\
\quad \text { Otros servicios de } \\
\text { reparación }\end{array}$ \\
\hline $\begin{array}{l}\text { la Mano de } \\
\text { Obra }\end{array}$ & $\begin{array}{l}\text { Servicios en Masa: } \\
\text { - Venta Detallista } \\
\text { - } \quad \text { Venta al por mayor } \\
\text { - } \quad \text { Ascuelas } \\
\quad \text { bancctos detallistas de la } \\
\quad \text { banercial }\end{array}$ & $\begin{array}{l}\text { Servicios Profesionales: } \\
\text { - } \quad \text { Medicina } \\
\text { - } \quad \text { Conogacía } \\
\text { - }\end{array}$ \\
\hline
\end{tabular}

Figura 3. Clasificación de los servicios por el contenido de sus recursos Fuente: Arias Aranda (2001) 


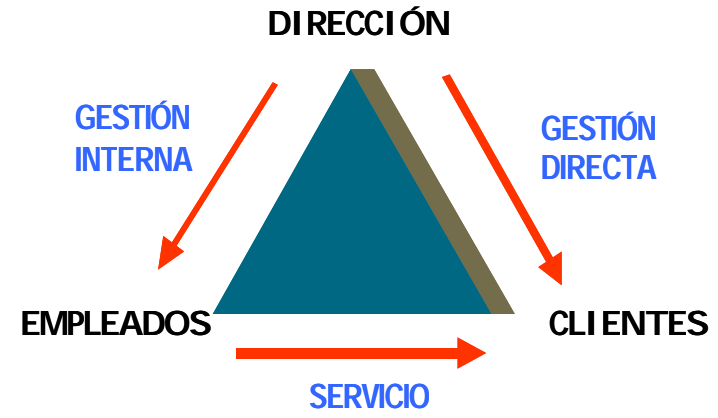

Figura 4. Cadenas de utilidades Fuente: Elaboración propia, 2006

servicio a entregar, mientras que en la segunda mide el ratio del costo de mano de obra respecto al costo del capital.

\section{Cadena de Utilidades}

La visión estratégica de las Operaciones de los Servicios están orientados a generar valor al servicio a través de las personas que son parte del servicio con el objetivo de satisfacer al cliente y exceder sus expectativas. Como el esquema mostrado en la figura 4.

Para que la alta dirección pueda satisfacer al cliente externo, primero tiene que satisfacer al cliente interno (empleados), la razón radica en la cadena de utilidades.
La cadena de utilidades vincula las utilidades de la empresa con la satisfacción de los empleados y de los clientes.

- Calidad interna: capacitación a los empleados, un ambiente de trabajo de calidad y un poderoso apoyo para quienes tratan con los clientes.

- Empleados satisfechos y productivos.

- Un valor agregado

- Clientes satisfechos y leales: clientes que refieren a otros clientes.

- Utilidades y crecimiento saludables.

Como se muestra en la figura 5.

\section{Estrategias de Crecimiento}

Sobre las estrategias de crecimiento que puede tener una empresa de servicios, debido muchas veces a la presión del mercado potencial y al deseo de proteger el concepto de servicios de las empresas competidoras mediante el alzamiento de barreras de entrada, las principales estrategias son:

- Servicio enfocado: prestación del servicio único y nuevo, generando alta calidad, pero bajas barreras para que aparezcan competidores.

- Red enfocada: empresas que desean ser accesibles a sus clientes ha de considerar la necesidad de localizarse en múltiples lugares manteniendo el control y la consistencia del servicio.

- Servicio aglomerado: las empresa de servicios con grandes instalaciones de carácter fijo

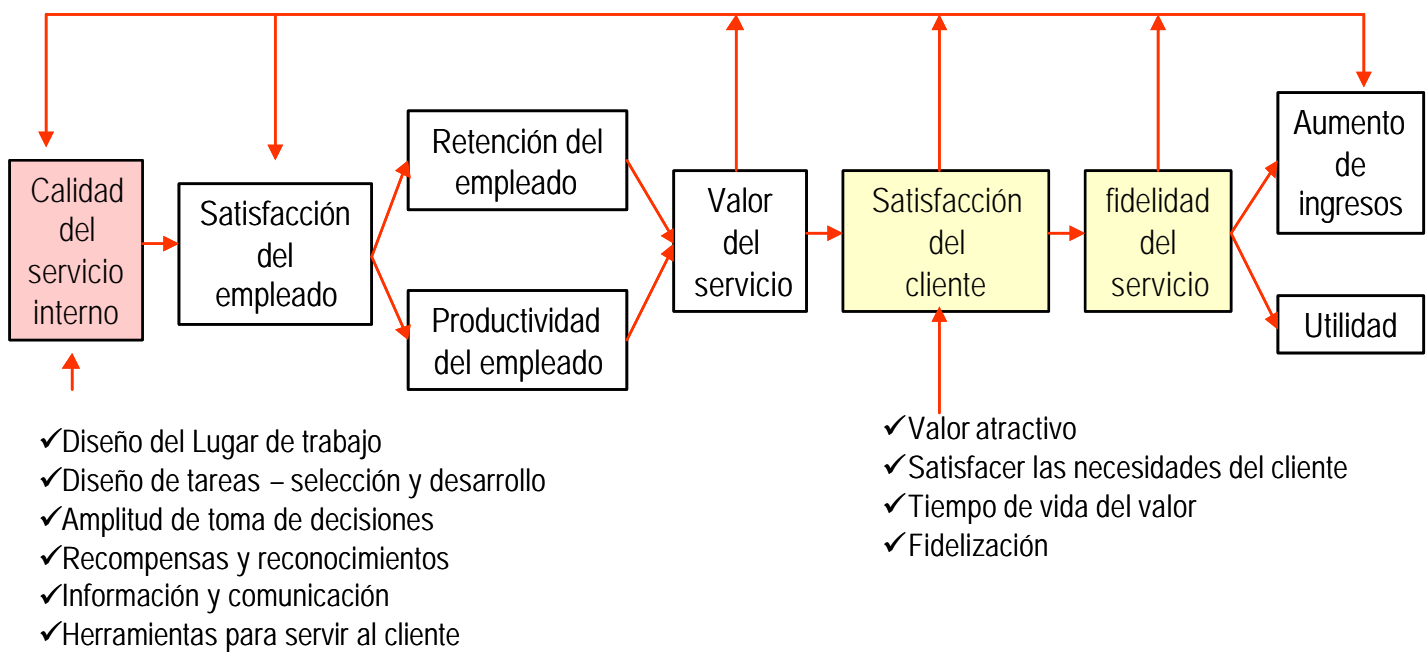

Figura 5. Vinculación de la cadena de utilidades con las utilidades de la empresa Fuente: Rivera y Huete (2004) 
Un solo servicio

Multiservicio

\begin{tabular}{|c|c|c|}
\hline $\begin{array}{l}\text { Una sola } \\
\text { localización }\end{array}$ & $\begin{array}{ll}\text { Servicio Enfocado: } \\
\text { - } \quad \text { Dentistas } \\
\text { - } \quad \text { Venta al por menor } \\
\text { - } \quad \text { Restaurante familiar }\end{array}$ & $\begin{array}{ll}\text { Servicio Aglomerado: } \\
\text { - } \quad \text { Gran Universidad } \\
\text { - } \quad \text { Gran empreseral de seguros }\end{array}$ \\
\hline $\begin{array}{c}\text { Múltiples } \\
\text { localizaciones }\end{array}$ & $\begin{array}{l}\text { Red enfocada: } \\
\text { - } \quad \text { Gran empresa de } \\
\text { - } \quad \text { Raquetería } \\
\text { - } \quad \text { restaurantes de comida } \\
\text { - } \quad \text { Cadenas hoteleras }\end{array}$ & $\begin{array}{l}\text { Red Diversificada: } \\
\text { - } \quad \text { Gran Caja de Ahorros } \\
\text { - } \quad \text { Gestora de tarjetas de } \\
\text { - } \quad \text { Grandes Consultores }\end{array}$ \\
\hline
\end{tabular}

Figura 6. Estrategias de expansión Fuente: Arias Aranda (2001)

deciden, en ocasiones , maximizar su potencial diversificando sus servicios.

- Red diversificada: empresas de servicios que crecen a través de adquisiciones, en la que tienenquecombinarestrategias demultilocalización con estrategias de multiservicios.

Diseño del subsistema de operaciones de servicios

En este apartado se incluye la selección y el diseño del servicio, la gestión de la capacidad, localización o la distribución de planta.

\section{Diseño del servicio}

Los clientes de las empresas de servicios obtienen beneficios y satisfacciones de los propios servicios y de la forma como se prestan esos servicios. La forma en que operan dichos servicios es algo relevante. Los sistemas de servicios que operan eficiente y efectivamente, pueden dar comercialmente un considerable apalancamiento y ventaja promocional. Es claro que la operación uniforme de un servicio ofrece ventajas competitivas, especialmente cuando la diferenciación entre productos puede ser mínima. Es decir, existirán ventajas si se funciona a tiempo, si no se exige demasiado a los clientes, 0 , entregan lo prometido. Asegurar que los sistemas de servicios funcionen eficiente y efectivamente, tradicionalmente es una tarea de la Dirección de Operaciones.

Por ello es importante tener un el diseño del servicio, como se muestra en la figura 7.

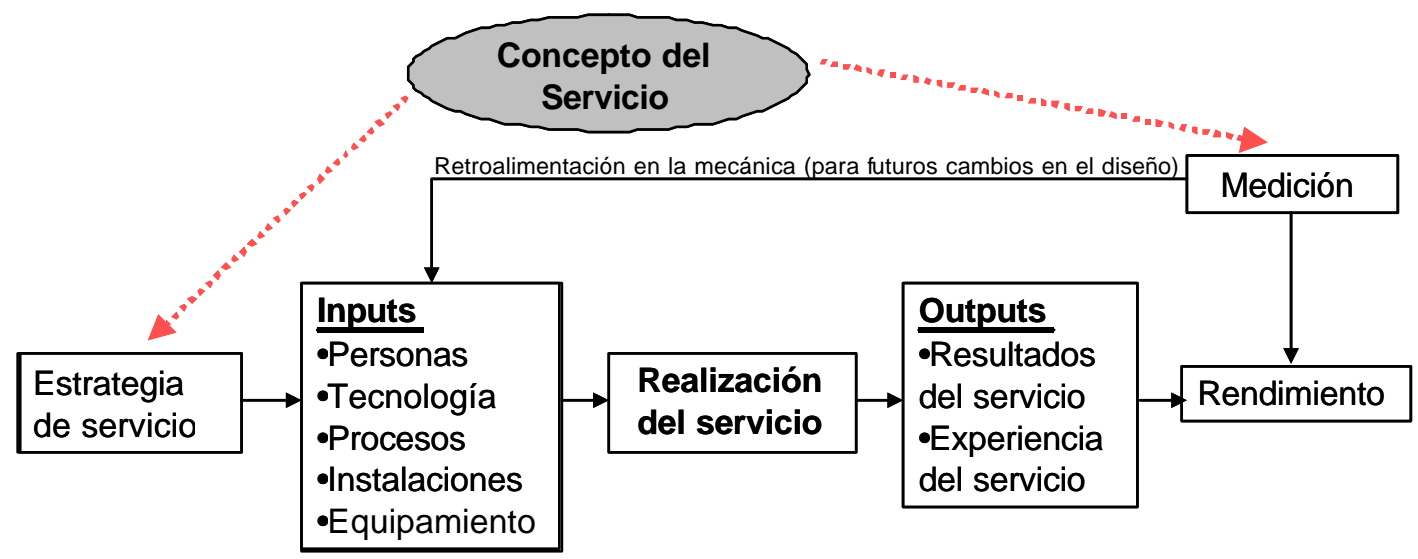

Figura 7. Estrategias de expansión Fuente: Arias Aranda (2001) 
El personal puede mitigar la descomposición del sistema, hasta cierto punto, prestando atención placentera y considerada a los problemas de los clientes. Unas instalaciones físicas agradables pueden suavizar el contratiempo de esperar más tiempo del previsto. Pero no pueden compensar totalmente las ineficiencias y las fallas del sistema. Qué tan bien funciona el sistema general, sus procedimientos y políticas, la participación del cliente en el proceso, el grado de estandarización del sistema; todas estas son preocupaciones de la dirección de operaciones.

Algunas de las dificultades de la Dirección de Operaciones para el diseño de los Servicios consiste en: establecer los objetivos, utilización de la capacidad, la participación de la gente en el proceso, control de calidad y en la aplicación del concepto de sistema. Una explicación de cada uno de estos puntos es:

Establecer objetivos en los sistemas de servicios: En algunos sistemas de servicios no se pueden utilizar medidas convencionales como utilidades y retorno sobre la inversión, se ocupan métodos alternos. Por lo tanto, en los servicios sin ánimo de lucro y el sector de servicios sociales, puede ser difícil y complejo establecer objetivos, a nivel general y a nivel de unidad operativa. Normalmente los objetivos para este tipo de servicios tendrán que incorporar medidas del nivel y calidad del servicio que en algunas partes se suministran, y éstas plantean dificultades.

Utilización de la capacidad: La intangibilidad de los servicios significa que hay limitaciones para la creación de inventarios, aunque naturalmente la gente y sus habilidades se pueden inventariar por acumulación de trabajo, y las instalaciones se pueden inventariar para ofrecer capacidad extra en caso necesario. Generalmente en los servicios lo que no se usa o está inactivo se pierde y no se puede emplear para llenar ninguna sobrecarga que pueda haber en el futuro. Una decisión fundamental en la administración de un servicio es qué nivel de capacidad se suministrará. Demasiada hace la operación antieconómica; muy poca puede causar dificultades en la prestación del servicio y malestar en el cliente debido a la ineficiencia y pérdida de negocios. La mayor parte de las organizaciones de servicios no puede tener algo así como una "Inventario de servicios".

Participación del cliente en el proceso del servicio:Como se dijo anteriormente, los clientes con frecuencia juzgan la calidad de un servi- cio y quedan satisfechos con él a través de la calidad de la relación con los empleados del servicio. Evidentemente los modales de los empleados, la calidad de su capacitación y su conocimiento de los servicios disponibles son influencias importantes sobre dichas satisfacciones. Pero a la larga los empleados operan sistemas. Los empleados pueden hacer todo lo que les sea posible para ayudar a los clientes, pero no pueden compensar totalmente sistemas malos, ineficientes e injustos. En la dirección de operaciones hay transacción entre la gente y los sistemas. Quitar discreción a los empleados de un servicio que opera en un sistema puede tener buen sentido económico y dar como resultado un servicio más estandarizado. También puede significar servicio más económico, tal vez de una calidad más uniforme. Sin embargo, eso sólo se puede lograr a costa de la satisfacción del empleado. El diseño del sistema del servicio debe tomar en cuenta sus reacciones y su motivación: los clientes participan en el proceso de producción, por lo tanto, ellos también son un insumo para esta.

Control de calidad: Otra dificultad es el control de calidad. Muchos principios de control de calidad aplicables a manufactura, se pueden aplicar también a los servicios. Algunos de estos son el control de calidad incluye a todo el mundo en una operación de servicio en tareas visibles y no visibles; es necesario utilizar sistemas para identificar fallas de calidad, recompensar éxitos y ayudar con mejoras; el control de calidad puede mejorarse reemplazando gente con máquinas, especialmente en tareas de rutina.

El concepto de sistemas de servicios. El concepto de sistemas se usa ampliamente en la dirección de operaciones para moldear la naturaleza fundamental del proceso de conversión en manufactura (es decir, existe una secuencia lógica con insumos de materias primas que pasan a través de un proceso de conversión en el cual se aplican tecnologías, se agrega valor y se producen los bienes terminados). Sin embargo, la aplicación de este modelo es más difícil en algunos sistemas de servicios debido a que las distinciones entre insumos, conversiones y resultados son menos claras.

Planificación, programación y control de servicios

En esta parte se tocará aspectos de la planificación, programación y control de las operaciones y de la capacidad, la gestión de los inventarios, la medición de la calidad de servicios o la gestión de proyectos. 


\section{Diseño del proceso de Servicio}

La dirección de operaciones se refiere a la planeación, programación y control de este proceso de conversión de recursos. El concepto útil es importante; para efectos del proceso es agregar utilidad o valor por encima de todos los costos en los cuales se incurre para obtener insumos del sistema y emprender el proceso de transformación.

Los sistemas operativos de servicios pueden ser clasificados, esta clasificación puede ser de varias maneras las que están relacionadas con el tipo de proceso y con el grado de contacto.

Según el tipo de proceso, los tres procesos aplicables a las empresas de servicios son:

Operaciones en línea: En una operación en línea hay una secuencia organizada de operaciones o actividades. El servicio se produce siguiendo esta secuencia. El alto grado de interrelación entre los diferentes elementos de una operación de línea significa que el rendimiento general está limitado por el rendimiento en el eslabón más débil del sistema y pueden surgir demoras en la entrega del servicio completo. Igualmente tiende a ser un tipo de proceso relativamente inflexible, aunque las tareas del proceso se puedan especializar y rutinizar dando un resultado más rápido. Este proceso es más conveniente en empresas de servicios con altos volúmenes de demanda continua de clases de servicios relativamente estándares.
Operaciones combinadas: Una operación combinada produce una variedad de servicios que utilizan diferentes combinaciones y secuencias de actividades. Los servicios se pueden ajustar para satisfacer diversas necesidades de los clientes y ofrecer un servicio solicitado. Si bien la flexibilidad es una ventaja clave de este tipo de sistema, puede resultar más difícil de programar, más difícil de sustituir capital por mano de obra en el sistema y puede ser más difícil de calcular la capacidad del sistema.

Operaciones intermitentes: Lasoperaciones intermitentes se refieren a los proyectos de servicios que son una vez o infrecuentemente repetidos. La escala de esos proyectos hace que su administración resulte una tarea compleja. Esos proyectos ofrecen un campo apropiado para la fácil transferencia de muchas técnicas de control y programación. La escala e infrecuencia de dichos proyectos los diferencia de las operaciones de línea y combinadas.

Otra clasificación usada en la Taxonomía para el diseño del procesos de servicio aporte de Fitzsimmons y Fitzsimmons (1997), en la cual clasifica los procesos de servicio usando el concepto de divergencia, el objeto a través del cual la actividad del servicio es directa, y el grado de contacto con el cliente, es decir baja divergencia ( servicios estandarizados) y alta divergencia (servicios personalizados), como se muestra en el cuadro 1.

Cuadro 1. Clasificación de los servicios usando el concepto de divergencia

\begin{tabular}{|c|c|c|c|c|c|c|c|}
\hline & \multicolumn{3}{|c|}{$\begin{array}{c}\text { Baja Divergencia } \\
\text { (servicios estandarizados) }\end{array}$} & \multicolumn{3}{|c|}{$\begin{array}{c}\text { Alta divergencia } \\
\text { (Servicios personalizados) } \\
\end{array}$} \\
\hline & & $\begin{array}{l}\text { Procesamiento } \\
\text { de bienes }\end{array}$ & $\begin{array}{l}\text { Procesamiento } \\
\text { de Información e } \\
\text { Imágenes }\end{array}$ & $\begin{array}{l}\text { Procesamiento } \\
\text { de personas }\end{array}$ & $\begin{array}{l}\text { Procesamiento } \\
\text { de bienes }\end{array}$ & $\begin{array}{l}\text { Procesamiento } \\
\text { de Información e } \\
\text { Imágenes }\end{array}$ & $\begin{array}{l}\text { Procesamiento } \\
\text { de personas }\end{array}$ \\
\hline \multicolumn{2}{|c|}{ Sin contacto con el cliente } & Lavandería & $\begin{array}{l}\text { Procesos de } \\
\text { facturación con } \\
\text { tarjeta de crédito }\end{array}$ & & $\begin{array}{l}\text { Autoreparar } \\
\text { Adaptar un juego }\end{array}$ & $\begin{array}{l}\text { Programación de } \\
\text { sistemas } \\
\text { Diseño de casas }\end{array}$ & \\
\hline \multicolumn{2}{|c|}{$\begin{array}{l}\text { Contacto indirecto con el } \\
\text { cliente }\end{array}$} & & $\begin{array}{l}\text { Servicio a } \\
\text { domicilio de } \\
\text { comida rápida. }\end{array}$ & & & $\begin{array}{l}\text { Supervisión del } \\
\text { aterrizaje por un } \\
\text { controlador } \\
\text { aéreo }\end{array}$ & \\
\hline \multirow[t]{2}{*}{$\begin{array}{l}\text { Contacto } \\
\text { directo } \\
\text { con el } \\
\text { cliente }\end{array}$} & $\begin{array}{l}\text { No hay } \\
\text { interacción } \\
\text { entre el } \\
\text { cliente y el } \\
\text { personal } \\
\text { (Auto } \\
\text { servicio) }\end{array}$ & $\begin{array}{l}\text { Operación de } \\
\text { máquinas } \\
\text { dispensadoras } \\
\text { (vending } \\
\text { machine) } \\
\text { Armado de } \\
\text { muebles pre- } \\
\text { fabricados }\end{array}$ & $\begin{array}{l}\text { Sacar dinero del } \\
\text { cajero } \\
\text { automático }\end{array}$ & $\begin{array}{l}\text { Operar un } \\
\text { elevador }\end{array}$ & $\begin{array}{l}\text { Mostrar alimento } \\
\text { en una cena } \\
\text { buffet } \\
\text { Empacar } \\
\text { comestibles }\end{array}$ & $\begin{array}{l}\text { Documentar la } \\
\text { historia clínica } \\
\text { Buscar } \\
\text { información en } \\
\text { una biblioteca }\end{array}$ & $\begin{array}{l}\text { Conducir un auto } \\
\text { rentado }\end{array}$ \\
\hline & $\begin{array}{l}\text { Interacción } \\
\text { entre cliente } \\
\text { y el } \\
\text { personal }\end{array}$ & $\begin{array}{l}\text { Sirviendo la } \\
\text { comida en un } \\
\text { restaurante } \\
\text { Car washing }\end{array}$ & $\begin{array}{l}\text { Transacciones } \\
\text { en un Banco }\end{array}$ & $\begin{array}{l}\text { Transporte } \\
\text { público }\end{array}$ & $\begin{array}{l}\text { Servicios de } \\
\text { limpieza a } \\
\text { domicilio }\end{array}$ & $\begin{array}{l}\text { Pintado de } \\
\text { retrato }\end{array}$ & Peluquerías \\
\hline
\end{tabular}

Fuente: Adaptado de Fitzsimmons y Fitzsimmons (1998) 


\section{Calidad de Servicio}

Atender a los clientes con excelencia "CALIDAD", con la cantidad mínima de recursos. ¿Cómo?

- Capacitar mejor a los empleados.

- Estandarizando los procedimientos.

- Introducir tecnología: ahorro de tiempo y dinero.

Debe tomarse en cuenta que al reducir costo también se puede reducir calidad en el servicio. Las Empresas de servicios buscan satisfacer a sus clientes como su principal objetivo.

Aquí se tienen algunas acciones comunes:

Visión de Servicio: La calidad de servicio es la clave del éxito si se quiere satisfacer al cliente. Ven el servicio como parte integral del futuro de la organización, no como algo periférico. Creen fundamentalmente que un servicio es la mejor estrategia del éxito y la mejor estrategia para generar beneficios. Al margen de los objetivos del mercado, del paquete de servicios o de las políticas de precio seguidas, los líderes de la satisfacción al cliente conciben la calidad de servicio como la base de la competencia. Desde cualquier ángulo que se mire, la idea del servicio, constituye la idea fundamental.

Altos estándares de calidad: Una verdadera dirección de la calidad aspira a un servicio que se convierta en algo legendario; son conscientes de que un buen servicio puede no ser lo suficientemente bueno como para diferenciar su empresa de las demás. Los directivos del servicio están interesados en los detalles y matices del servicio, ven oportunidades en pequeñas acciones que los competidores podrían encontrar triviales. Creen que la forma en que una empresa sepa llevar los pequeños detalles define el estilo con que se llevarán los grandes (detalles). También creen que las pequeñas cosas añadidas en beneficios del usuario marcan la diferencia.

Liderazgo sobre terreno: Dirigen las operaciones a donde está la acción, en vez de dar órdenes desde su escritorio. Están siempre visibles para sus empleados; siempre entrenando, corrigiendo, persuadiendo, hablando, sermoneando, observando, preguntando y escuchando. Ponen énfasis en mantener una comunicación de dos vías con su personal. Utilizan un enfoque de permanente motivación para conseguir un clima de trabajo en equipo en la organización. Incitan a las unidades operativas con el fin de alcanzar la perfección en el servicio (y no sólo al empleado como individuo) y utilizan la influencia de sus ejecutivos para lograr que el equipo se congregue con frecuencia en reuniones, juntas y celebraciones.

Integridad: Una de las características esenciales del liderazgo del servicio es la integridad personal. La más importante cualidad del liderazgo es el valor que se asigna a hacer lo correcto, aun cuando no sea conveniente o sea muy costoso. Los I íderes asignan un valor extra al hecho de ser justos, consistentes y fiables y como resultado ganan la confianza plena de sus colaboradores. Los líderes en el servicio reconocen la imposibilidad de establecer una actitud orientada al servicio en una empresa cuya dirección carece de integridad. Reconocen la estrecha relación que existe entre la calidad del servicio y el orgullo que sienten los empleados y entienden que esa actitud de orgullo se debe, en parte, a la percepción que tienen los empleados de una dirección justa.

Las principales clases de servicios que otorgan satisfacción: Hay un número infinito de opciones de servicio que ofrecen las empresas, por lo tanto en la presente calificación se refiere sólo a lo más esencial. La idea general de otorgar satisfacción es agregándole valor al servicio, y una forma es con la calidad del servicio, como se muestra en el siguiente esquema:

Servicio de prolongación de la vida útil: Los servicios más importantes ofrecidos por los proveedores, en función del dinero gastado, son los destinados a mantener el producto en condiciones satisfactorias de operación, durante un tiempo prolongado. Su propósito es brindar al cliente una mayor satisfacción con el servicio, y más aún, evitar una situación que lo obligue a descartar un ítem costoso por falta de una pieza o de un técnico que pueda repararla.

La creciente complejidad de los productos, la reacción de los clientes, la competencia y el reconocimiento de que los servicios de mante-

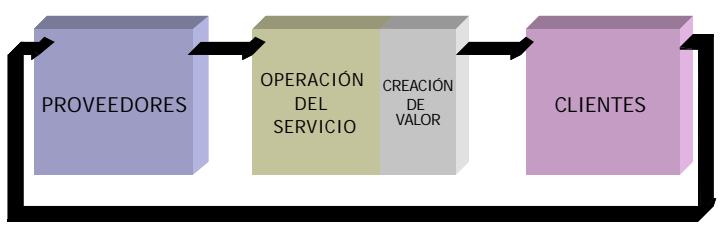

Figura 8. Proceso de creación de valor en los servicios Fuente: Elaboración propia, 2006 
nimiento y reparación pueden resultar altamente provechosos, han contribuido a su enorme expansión en las últimas dos décadas.

Servicio de disminución de trabajo: Este servicio tiende a facilitar la compra del cliente haciéndola más cómoda y fácil. Apela a todos los tipos de compradores, pero se ofrece más a los consumidores últimos, a nivel minorista.

Ejemplo: Dar información al cliente potencial, ya sea en mostrador o por medio de vendedores; contestar a diversas preguntas sobre los artículos. Se pueden idear servicios especiales, en este sentido por ejemplo: bolsas de papel, climatización del local, servicio de entrega a domicilio (suele ser uno de los más importantes). Servicios como estos son básicos y marcan una gran lealtad y satisfacción a los clientes.

Otros servicios, para evitar trabajo:

- Toma de pedido por teléfono

- Estacionamiento de automóviles (la falta de estacionamiento puede alejar a los clientes, ya sea actuales o potenciales).

Servicio de financiamiento: El servicio de financiamiento puede ser beneficioso para todo tipo de clientes.

Por ejemplo, el diseño de un servicio de crédito requiere decisiones acerca de sus límites, tipos de interés, plazos de amortización y selección de clientes. Los créditos son ejemplos claros de servicios utilizados por los clientes, pero no por todos, también existe un abuso del crédito en términos de usura que minará la satisfacción del consumidor por los cobros excesivos.

Servicio de incremento de ventas: La capacitación de los vendedores y distribuidores, con respecto a las técnicas de ventas, constituirán probablemente el más valioso de los incrementos de ventas. Quizás este servicio es uno de los más importantes a la hora de medir satisfacción por el contacto que el personal tiene con los clientes.

Cadena de suministro y logística

En esta parte se hace referencia a las redes de suministro, las estrategias y canales de distribución, el diseño de rutas de vehículos o programación de vehículos. Su aplicación es semejante a la estudiada en la Dirección de Operaciones específicamente en transportes.

C ON C L U S I O NE S

Existe un leve crecimiento de la investigación en DOS, pero todavía insignificante, respecto a la relevancia del tema y más en países en vías de desarrollo, por lo que algunos temas de la Dirección Operaciones que no son estudiadas para empresas de servicios.

El uso de técnicas y métodos de dirección de operaciones en empresas de servicios genera aportes significativos según los estudios revisados. Sin embargo, la ausencia de investigación sobre los conceptos de servicio, en un esquema general impiden mayores aplicaciones de Dirección de Operaciones en empresas de servicios.

Este campo vislumbra algunas futuras líneas de investigación que pueden ser: Estado de la Investigación en DOS en el periodo 2003 - 2006, Magnitud y aporte de empresas de servicios en los países en vías de desarrollo, Concepto de servicios como definición general, Manufactura de bienes con valor agregado de servicios, Servicios completamente virtuales, Investigación en las actividades de servicios profesionales, entre otros.

Asimismo, plantea algunas interrogantes que se quedan sin responder: ¿Los clientes compran un concepto o una especificación?, ¿Los clientes consume parte del servicio o se enmarcan en una visión general?, ¿Cómo el concepto del servicio puede usarse para crear lineamientos organizacionales?, ¿Se utiliza la Dirección de Operaciones en las empresas de Servicios de los diversos países de Latinoamérica?, ¿Puede el concepto de servicio ser usado para conducir la ventaja competitiva?, ¿Qué característica del servicio determina un apropiado desempeño?, ¿Cuál es el impacto de relacionar las medidas de desempeño del servicio sus componentes del servicio (personas, tecnología, procesos...)?, ¿Cómo podemos identificar las brechas entre las expectativas o percepciones que queremos generar a los clientes(Intenciones estratégicas) y las reales(el concepto de nuestro servicio)?.

REFERENCIAS BIBLIOGRÁFICAS

1. Arias Aranda, D. (2001). La estrategia de Operaciones en la empresas de servicios: un marco teórico, Revista de dirección, organización y administración de empresas (Revista CEPADE), Nro. 25. pp. $134-137$.

2. De Burgos, J.; Céspedes, J. J.; Alvarez, M. J. (2003). Dirección de Operaciones de servicios : los hoteles españoles, Revista Europea de Dirección y Economía de la empresa, Vol. 12 , Nro. 1, pp 83-98.

3. Goldstein, S. M.; Johnston, R.; Duffy, J.; Rao, J. 
(2002). The Service Concept: The Missing Link In Service Design Research?, Journal of Operations Management, vol. 20, pp. $121-134$

4. Gonzales Zamora, M.; Domínguez Machuca, J. A.; Aguilar Escobar, V.G (2003). Un estudio sobre la investigación en dirección de Operaciones en empresas de servicios, Documento de Trabajo de la Universidad de Sevilla. G.I.D.E.A.O. Facultad de Ciencias Económicas y Empresariales

5. Fitzsimmons, J.A.; Fitzsimmons, M.J. (1998). Service Management: Operations, Strategy, and Information Technology. New York, NY: Irwin/ Mc Graw Hill.

6. Kotler, P. (1993). Principios de Marketing. Prentice Hall, 7ª Edición.
7. Lovelock, C.; Reynoso, J.; D’Andrea, G.; Huete, L. (2004). Administración de servicios. México: Pearson Hall, $1^{\text {a }}$ Edición.

8. Lescano Duncan, L. (2001). La disciplina del servicio. Lima: Universidad del Pacifico. Centro de Investigación, 1aㅡ Edición.

9. Pancorvo Corcuera, J. (2001). Organizaciones de Servicios. Piura: PAD- Escuela de Alta Dirección. Universidad de Piura. $1 \stackrel{\text { a }}{ }$ Edición.

10.Rivera, J.; Huete, L. M. (1997). Empresas de Servicios: Un Mundo de Posibilidades. Barcelona: Biblioteca IESE de Gestión de Empresas.

11.Stanton, W. J. (1992). Fundamentos de Marketing - McGraw Hill, 9ª Edición. 